\title{
Testing The Efficacy of a Couple-Focused, Tailored eHealth Intervention for Symptom Self-Management Among Men with Prostate Cancer and Their Partners: The Study Protocol
}

\section{Lixin Song ( $\sim$ lsong@unc.edu )}

School of Nursing, University of North Carolina (UNC), Chapel Hill, NC https://orcid.org/0000-0001-8286-319X

Matthew E. Nielsen

Lineberger Comprehensive Cancer Center, UNC, Chapel Hill, NC; School of Medicine, UNC, Chapel Hill, NC

\section{Ronald C. Chen}

University of Kansas Medical Center

Christine Rini

Northwestern University, Cancer Survivorship Institute and Department of Medical Social Sciences, Chicago

Thomas C. Keyserling

School of Medicine, UNC, Chapel Hill, NC

\section{Eno Idiagbonya}

School of Nursing, University of North Carolina (UNC), Chapel Hill, NC https://orcid.org/0000-0002-5714-0640

\section{Gail P. Fuller}

School of Nursing, University of North Carolina (UNC), Chapel Hill, NC

\section{Laurel Northouse}

School of Nursing, University of Michigan, Ann Arbor, MI

\section{Mary H. Palmer}

School of Nursing, University of North Carolina (UNC), Chapel Hill, NC

\section{Xianming Tan}

Lineberger Comprehensive Cancer Center, UNC, Chapel Hill, NC; Gillings School of Global Public Health, UNC, Chapel Hill, NC

\section{Study protocol}

Keywords: Prostate cancer, caregiver, eHealth, symptom management, quality of life, randomized clinical trial, social support, stress, coping, health behavior

Posted Date: August 2nd, 2021

DOI: https://doi.org/10.21203/rs.3.rs-685875/v1

License: (c) (7) This work is licensed under a Creative Commons Attribution 4.0 International License. Read Full License 
Version of Record: A version of this preprint was published at Trials on January 4th, 2022. See the published version at https://doi.org/10.1186/s13063-021-05948-5. 


\section{Abstract}

Background: Men with localized prostate cancer often experience urinary, sexual, bowel, and hormonal symptoms, general distress, pain, fatigue, and sleep disturbance. For men in an intimate relationship, these symptoms disrupt couples' relationships and intimacy. The symptoms also reduce quality of life of both men and their partners, who are often their primary caregivers. Management of the negative effects of cancer and its treatment is a significantly underaddressed supportive care need for these men and their intimate partners. To address these unmet supportive care needs, our interdisciplinary team developed and pilot tested the usability and feasibility of an evidence-based, couplefocused, tailored eHealth intervention, "Prostate Cancer Education \& Resources for Couples" (PERC). Based on the adapted stress and coping theoretical framework and developed with stakeholder involvement, PERC aims to improve quality of life for both men and their partners by enhancing their positive appraisals, self-efficacy, social support, and healthy behaviors for symptom management.

Methods: We will test the efficacy of PERC using a population-based, geographically and demographically diverse cohort in a randomized controlled trial. Primary Aim: Assess if patients and partners receiving PERC will report greater improvement in their cancer-related quality of life scores than those in the control group (usual care plus the National Cancer Institute prostate cancer website) at 4, 8, and 12 months post-baseline. Secondary Aim: Test if patients and partners in PERC will report significantly more positive appraisals and higher levels of coping resources at follow-ups than those in the control group. Exploratory Aim: Determine if patient race and ethnicity, education, type of treatment, or couples' relationship quality moderate the effects of PERC on patient and partner QOL at follow-ups..

Discussion: This study will provide a novel model for self-managing chronic illness symptoms that impact couples' relationships, intimacy, and quality of life. It addresses the National Institute of Nursing Research's goal to develop and test new strategies for symptom self-management to help patients and caregivers better manage their illness and improve quality of life. It also responds to calls for programs from the Institute of Medicine and American Cancer Society to address treatment-related effects and improve survivors' QOL..

\section{Trial registration: CT.gov ID: NCT03489057}

\section{Introduction}

\section{Background and rationale $\{6 a\}$}

Prostate cancer is the most frequently diagnosed non-skin cancer in men in the U.S., with 191,930 new cases in 2020. (1) Approximately $92 \%$ of diagnosed men have local or regional disease.(2) They are treated with curable intent (prostatectomy or radiation therapy, with or without hormonal therapy), but they often experience distressing treatment-related urinary, sexual, bowel, and hormonal symptoms (e.g., urinary incontinence and urinary urgency, erectile dysfunction, diarrhea, and hot flashes) that linger for months or years.(3-7) These symptoms also lead to emotional distress, fatigue,(8) pain and sleep disturbance, $(5,9)$ and reduced quality of life $(\mathrm{QOL}),(7,10)$ all of which can be addressed by self-management. With an approximately $97.5 \% 5$-year relative survival rate and median age of 66 at diagnosis,(11) men with localized prostate cancer are likely to survive for many years and die from other illnesses.(12-15) [Note: Five-year relative survival rates describe the percentage of patients with a disease alive five years after the disease is diagnosed, divided by the percentage of the general population of corresponding sex and age alive after five years]. Psychosocial behavioral self-management strategies (e.g., healthy diet and physical activities) are effective for reducing general symptoms, $(16,17)$ decreasing overall and cancer-specific mortality,(18$20)$ and improving QOL among aging populations (including cancer patients).(17, 21, 22) 
For men who are in an intimate relationship, partners are a critical source of support. They are often the primary caregiver and they play an important role in seeking and gathering information besides providing tangible care and emotional support.(23) Prostate cancer and treatment-related symptoms also reduce partners' QOL,(24, 25) leading to disruption in couples' intimacy and relationships.(26-29) The adverse effect of symptoms on the QOL of partners may be as great or greater than on the QOL of the patient diagnosed with prostate cancer. $(24,25)$ Addressing the needs of both the men and their partners is therefore of particular concern because the QOL of both is significantly related; $(25,30)$ each one's unmet needs impact their own and each other's QOL. $(30,31)$ The national agenda of the American Cancer Society (32) and National Comprehensive Cancer Network $(33,34)$ have included calls for programs to manage the physical and psychosocial effects of prostate cancer and its treatment, promote healthy behaviors for survivors and their families, and ultimately, improve their QOL.

To address the unmet needs for men and their intimate partners, our interdisciplinary team has developed a tailored, couple-focused eHealth intervention called Prostate Cancer Education \& Resources for Couples (PERC). PERC was developed based on contributions from stakeholders (survivors, partners, and oncology care providers),(35) findings from efficacious interventions with prostate cancer survivors and partners, ${ }^{16,17}$ and empirical evidence. $(32,34,36-38)$ Guided by an adapted stress and coping theoretical framework, $(39,40)$ PERC aims to improve QOL for both men undergoing treatment and their partners by enhancing positive appraisals of illness and boosting self-efficacy, social support from multiple sources, and healthy behaviors for symptom management. PERC uses eHealth technologies to dramatically increase couples' ability to access post-treatment supportive care whenever and wherever they feel comfortable accessing it. We tested the usability and feasibility of PERC and refined PERC in two pilot studies (UNC Cancer Prevention and Control Intervention Research Pilot Grant Award and UNC LCCC Population Sciences Developmental Research Award, PI: Song for both awards).(35)

\section{Objectives $\{7\}$}

We are currently conducting a randomized clinical trial (RCT) to test the efficacy of PERC (R01 NR016990, PI: Song; CT.gov ID: NCT03489057, IRB: 17-0482). We plan to achieve the following specific aims.

Primary Aim: Assess the efficacy of PERC for improving QOL among men undergoing treatment and their intimate partners. We hypothesize that men and their partners receiving PERC will report greater improvement in their cancerrelated QOL scores than those in the control group (usual care plus the National Cancer Institute (NCl) prostate cancer website) at 4-, 8-, and 12-months post-baseline.

Secondary Aim: Test the effects of PERC on symptom appraisals and coping resources. We hypothesize that men and their partners receiving PERC will report greater improvement in secondary outcomes, positive appraisals of illness and coping resources (i.e., self-efficacy in symptom management, greater social support, and use of more healthy behaviors) at follow-ups than those in the control group.

Exploratory Aim: Examine if men undergoing treatments' race/ethnicity, education, type of cancer treatment, or couples' relationship quality at baseline moderate the effects of PERC on patient and partner cancer related QOL at follow-ups.

\section{Trial design $\{8\}$}

This is a population-based, statewide, two-arm, parallel groups RCT to test the efficacy of PERC to improve the QOL of men with prostate cancer who are post-treatment and their intimate partners. 


\section{Methods: Participants, Interventions And Outcomes}

\section{Study setting $\{9\}$}

We recruit patient-partner couples using the North Carolina Central Cancer Registry Rapid Case Ascertainment (RCA). The RCA uses an accelerated process to capture new cases within a week of diagnosis. We conduct data collection surveys via telephone. During COVID-19, we added online surveys in response to participants' need for flexible scheduling for data collection. The intervention is conducted online or via telephone, according to participants preference.

\section{Eligibility criteria $\{10\}$}

Men are eligible if they (1) are 40-75 years of age and within 16 weeks of completing initial curative-intent treatment for localized prostate cancer (as confirmed by medical record); (2) have no prior cancer within the past 2 years; (3) are not currently receiving treatment for a concurrent cancer (excluding non-melanomatous skin cancer); (4) experience prostate cancer-specific and/or general symptoms (as assessed by screening questions); and (5) have an intimate partner (male or female) who is willing to participate. Intimate partners must be 18 years or older and not have been diagnosed with cancer or received treatment for cancer within the past 12 months; this criterion ensures that couples can focus their efforts on managing prostate cancer. Patients and partners must be able to read and speak English and they cannot have severe cognitive impairment. We will exclude men who are waiting for their initial treatment or on active surveillance.

\section{Who will take informed consent? $\{26 a\}$}

Trained research assistants who are responsible for participant enrollment and survey completion.

\section{Additional consent provisions for collection and use of participant data and biological specimens \{26b\}}

We mail potential participants an introductory letter, a brochure, an opt-out letter (which they can mail back if not interested), and informed consent information. Our trained research assistants then call within two weeks of mailing of the materials to assess interest in participating, answer questions, and screen for eligibility. We use the same procedure to screen partners' eligibility after eligible men give permission for us to contact their partner. The research assistants obtain informed consent from men and their partners separately via telephone. All consents were audio recorded and saved in a password-protected encrypted folder. Participants can also choose to mail their signed informed consent forms back to the study team.

\section{Interventions}

\section{Explanation for the choice of comparators $\{6 \mathrm{~b}\}$}

We use usual care plus the $\mathrm{NCl}$ prostate cancer website as the control condition. In usual care, patients access a range of sources (e.g., health providers, handouts and books, and online) to satisfy their information needs. (41) To ensure that our usual care participants access evidence-based, guideline-adherent information, we also give them access to the $\mathrm{NCl}$ website (http://www.cancer.gov/cancertopics/types/prostate)_through the study website. The NCI website provides information about prostate cancer treatment options, research, causes, and statistics; coping resources that are not prostate cancer-specific; and support from non-providers via a toll-free phone and LiveHelp Online Chat about cancer-related questions, clinical trials, and quitting smoking. After logging in to the study website, usual care participants meet with the study educator at orientation using their preferred method (telephone or online) and then 
meet with the educator every four weeks via email. They receive general information about treatment and referral to the $\mathrm{NCl}$ website. Couples who fail to access the $\mathrm{NCl}$ website, as indicated by the web activity tracking data, receive automatic messages via their preferred communication method (email, text, phone, or mail) to remind them to visit the website.

\section{Intervention description $\{11 \mathrm{a}\}$}

In response to increasing the use of technology to access information (including by older adults) (42-45) and to promote and help uptake of PERC, we use eHealth technologies to improve access to the knowledge and skills that are critical and sensitive during care transitions for both patients and their partners. The PERC website includes three major sections: (1) 10 education modules with post-session assignments, (2) a moderated online Forum, and (3) a Resource Toolbox. We provide links to scientific publications in the Resource Toolbox to satisfy the learning needs of participants with high education levels. PERC uses clear, easy-to-read text; minimizes webpage navigation redirects; and incorporates audio-enhanced slides, videos, and graphics in each module to reach people at different reading and education levels and with different learning styles. The research staff provides ongoing technical support via telephone and online during 8am to 9pm Monday to Saturday. In-person visits can be arranged if needed.

PERC also facilitates informational, appraisal, and emotional support from partners, peers, and professionals during care transitions. PERC assists couple support between the patient and his partner through couple-focused modules and post-session assignments in which couples practice effective communication skills; share with each other personal perceptions of and experiences with different symptoms; and collaboratively develop strategies to minimize negative perceptions and effects of these symptoms on their lives. As strongly recommended by our pilot participants, (35) couples are encouraged to log in to PERC from the same computer so that they can work together, although they can use the program separately at their convenience.

Participants can obtain peer and professional support using the online Forum moderated by our trained nurse educator who has extensive knowledge about prostate cancer and experiences working with men with prostate cancer and their families. Participants can also receive professional support by scheduling monthly virtual meetings (and additional meetings as needed) with the educator, either online or via a computer-assisted telephone call. At the meetings, which last 15-30 minutes, the educator helps couples review their symptom and management, identifies goals to achieve, reviews the online modules if needed, troubleshoots any technical problems, and encourages couples to use the different components of the PERC program. These meetings consist of PERC orientation (week 1), completion of modules (weeks 2-14), and the final session (week 15).

\section{Criteria for discontinuing or modifying allocated interventions $\{11 \mathrm{~b}\}$}

Patients and their partners will become ineligible for further participation in this study if either or both receives a diagnosis of any type of cancer (e.g., breast, bowel, etc.) except for non- melanomatous skin cancer or develops a condition that prevents them from fully participating in study activities such as scheduling and completing surveys, or online or computer-assisted telephone call with the Nurse Educator. Participants will also be removed from the study if they decide to withdraw from the study voluntarily.

\section{Strategies to improve adherence to interventions $\{11 \mathrm{c}\}$}

We follow fidelity guidelines for health behavior research recommended by the NIH Behavior Change Consortium (46) and recommendations to ensure fidelity for mHealth interventions (47). Training: before initiating PERC, we trained the nurse educator about the program, the PERC website, and process monitoring, using a standardized protocol checklist developed and refined in our pilot studies. PERC Implementation: Use of mHealth 
technology ensures that PERC is presented consistently. The nurse educator documents their intervention activities in the administrative log and records their online meetings with participants. The PI and project coordinator meets with the nurse educator to review fidelity issues and to provide feedback and demonstrations; these meetings occur weekly or whenever any member of the research team notices a discrepancy between the educator's activity and the protocol. PERC receipt: Our built-in web activity tracking system assesses, and records participant use of PERC. Participants who fail to enter the website are reminded via email, phone, or text messages. PERC was designed to minimize complexity by using plain language, using a low reading level, and implementing simple website navigation. $\underline{\text { PERC }}$ Enactment: The research team provides technical support to help address and document any issues that prevent participants' use of PERC website. .

\section{Relevant concomitant care permitted or prohibited during the trial $\{11 \mathrm{~d}\}$}

Participants are informed that this supportive care intervention does not interfere with their clinical care for cancer and/or other comorbid conditions. We anticipate that this is a minimal risk study. The PERC and NCI websites provide state-of-science resources that participants can use at their convenience. We will also refer them to their treating clinicians should any serious adverse medical or psychological event happen. No post-trial care is needed.

\section{Provisions for post-trial care $\{30\}$}

Not applicable.

\section{Outcomes $\{12\}$}

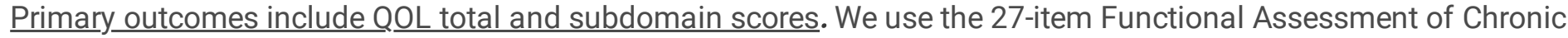
IIIness Therapy General Scale (FACT-G) to measure cancer-related QOL (48) and the physical, social, emotional, and functional domains of QOL.(49) Partners report their own cancer-related QOL using the partner's version of FACT-G with modified wording.(50)

Secondary outcomes include appraisal and coping resources. Appraisals of illness-patients' and partners' perceptions of the degree of threat associated with prostate cancer and related symptoms-are measured using a 20item version of the Appraisal Scales, abstracted from the 32-item patient version and the 27-item caregiver versions of the measure.(51) Responses are provided on a 5-point Likert response scale.(51, 52)

Self-efficacy is assessed by a 9-item modified version of the Lewis Cancer Self-Efficacy Scale, which measures confidence in managing prostate cancer symptoms. Responses are provided on a 10-point Likert scale. The partner version of the scale (53) evaluates partners' confidence in their own ability to manage prostate cancer symptoms.

Social support is assessed using the PROMIS Emotional, Informational and Instrumental Support Measures(5456) and the appraisal support subscale of the Social Support Questionnaire.(57)

For healthy behaviors, overall diet quality is assessed using the Dietary Risk Assessment (DRA) which is a brief food frequency dietary assessment.(58) Physical activity is assessed using the Measure of Older Adults' Sedentary Time (MOST) $(59,60)$ which includes time spent in sedentary behaviors such as watching TV, computer use, reading, transport and hobbies and total sedentary time.

Symptom outcomes. Prostate cancer-specific symptoms are measured using the Prostate Cancer Index Composite (EPIC-26).(61) For patients, we will calculate(61) EPIC's urinary irritability, urinary incontinence, bowel, sexual, and hormonal subscale scores. The partners complete a four-item EPIC (spousal version), which assesses how much of a problem the patients' bowel, hormonal, sexual, or urinary symptoms were for the partner. 
General symptoms. Patients and partners separately rate their own symptoms of pain, fatigue, sleep disturbance, (6265) cancer anxiety (66) and depression (67) using the PROMIS measures.

\section{Participant timeline $\{13\}$}

After informed consent and baseline assessments (T1, which occurs after completion of surgery or radiation), enrolled patient-partner couples are randomly assigned to PERC or enhanced usual care (usual care plus the $\mathrm{NCl}$ website) groups. After an intervention period that lasts approximately 15 weeks, participants complete three surveys to assess the short, intermediate, and long-term effects of PERC: at 4 months post-T1 (T2), 8 months post-T1 (T3), and 12 months post-T1 (T4).

\section{Sample size $\{14\}$}

We planned to enroll 250 patient-partner couples to achieve the study objectives. We calculated power for comparing our primary outcome (total QOL) using a standard approach for linear mixed models.(68) Because we will assess outcomes for patients and partners separately, we applied a Bonferroni-corrected, two-sided alpha of 0.025 to allow for separate overall tests for patients and partners.(68) Although dyadic data will be modeled simultaneously, this correction allows for the possibility that conclusions may differ for patients and partners. Based on our pilot test of PERC,(35) we assumed a common standard deviation for the total QOL scores of 15 points and a within-person correlation between repeated measurements of 0.75 . Also, we allowed for losing up to $7 \%$ of participants every 4 months, for a total attrition of $20 \%$ through 12 months.

\section{Recruitment $\{15\}$}

After receiving weekly reports of localized prostate cancer patients from North Carolina Central Cancer Registry Rapid Case Ascertainment (RCA), we contact patients' physicians by letter and give them two weeks to request that a patient not be approached for study inclusion. After the two-week window, we start to recruit using the procedure outlined above in the informed consent section.

\section{Assignment of interventions: allocation}

\section{Sequence generation $\{16 \mathrm{a}\}$}

After consented patients and partners independently complete the baseline survey, couples are randomized to PERC or the control group (usual care $+\mathrm{NCl}$ website) using a 1:1 ratio and stratified by type of treatment (surgery, radiation with or without hormonal therapy). Within each stratum, we use permuted block randomization with variable block size to generate allocation plan.

\section{Concealment mechanism \{16b\}}

Our study biostatistician prepares and centrally maintains the computer-generated random numbers and uploads the randomization plan to the REDCap system.

\section{Implementation \{16c\}}

Upon receiving the notice of a participant's completion of baseline surveys, the interventionist (i.e., a nurse educator) obtains from REDCap the group allocation for the enrolled couples and informs them of their assignment to initiate intervention activities. After randomization, participants are guided to access their assigned website $(\mathrm{PERC}$ or $\mathrm{NCI})$ via the study website using their assigned username and temporary password. 


\section{Assignment of interventions: Blinding}

\section{Who will be blinded $\{17 a\}$}

Our research team members (i.e., the Principal Investigator, the Co-Investigators except the biostatistician, and the data collectors) are blinded to participants' treatment allocation until the end of the study. The interventionist-the nurse educator-is not blinded but will not conduct surveys or interviews. The study participants are not blinded after they start to use their assigned eHealth program. We have no plan to unblind allocation to other team members before end of the study. We have separate staff meetings for data collectors and the interventionist to discuss any participantrelated issues and only use participants' study IDs in our discussion to maintain blinding during the study period.

\section{Procedure for unblinding if needed $\{17 \mathrm{~b}\}$}

N/A.

\section{Data collection and management}

\section{Plans for assessment and collection of outcomes $\{18 \mathrm{a}\}$}

Research staff (data collectors) collect study data using a telephone survey at baseline (upon enrollment, T1) and at 4, 8 , and 12 months post-T1. The data collectors are blinded to participants' group assignment. During COVID-19, we added online surveys in response to participants' need for flexible scheduling for data collection.

\section{Plans to promote participant retention and complete follow-up $\{18 \mathrm{~b}\}$}

Since retention in longitudinal studies can be a challenge, we use Northouse and colleagues' retention strategies (69) which were successful in our preliminary studies to maximize participant retention for both the PERC and control groups. We mail participants $\$ 20$ per person after they complete each survey, with additional incremental compensation to help motivate them for longer-term participation $(\$ 10, \$ 10$, and $\$ 30$ at the completion of the 4-, 8-, and 12-month post-baseline follow-ups, respectively). We also send participants retention gifts at 6 and 10 months. We also have regular team meetings to promptly address any issues that may negatively impact participant retention.

\section{Data management $\{19\}$}

Telephone surveys are scripted and audio-recorded, and data are entered simultaneously into the REDCap system, a secure, HIPAA-compliant database for data entry and management. The research team randomly checks at least 15$25 \%$ of the audio recordings against completed data for adherence to protocol, data completeness, and accuracy.

\section{Confidentiality \{27\}}

To protect the confidentiality of participant data, this study minimizes uses of hardcopy research records; the PI and the independent Safety Officer ensure all hardcopy records are saved in a locked cabinet in a locked private office. With most data and documents being electronic, the PI and the Safety Officer ensure that the identifiable and deidentified data and documents are saved separately in different project folders in the password-protected and encrypted, shared drive at the university, which is on a secure university server. Only authorized key study personnel will have access to the identifiable information.

The electronic data include survey recordings and recordings of meetings between the nurse educator and study participants for quality control, as well as the de-identified survey data, study progress data and documents, and web activity tracking data. The PI and the Safety Officer ensure that these data are tracked using study ID with no 
identifiable information attached. As a part of the university network and complying with university security regulations, we work closely with IT staff to ensure both security and efficiency for the study. Adverse event reports and annual summaries will not include information that can be used to identify participants. Each will include study IDs only.

\section{Plans for collection, laboratory evaluation and storage of biological specimens for genetic or molecular analysis in this trial/future use $\{33\}$}

This trial does not involve collection, laboratory evaluation, or storage of biological specimens for genetic or molecular analysis.

\section{Statistical methods}

\section{Statistical methods for primary and secondary outcomes $\{20 \mathrm{a}\}$}

Primary analyses will include all randomized participants, analyzed in the arm to which they are randomized, regardless of the extent of intervention received (intention-to-treat). Primary Aim: To assess the efficacy of PERC for improving $Q \underline{Q} \underline{\mathrm{OL}}$, we will compare the longitudinal mean change in overall QOL between groups using analysis of covariance (ANCOVA), conducted using linear mixed models. Data for patients and partners will be fit together in the same model (accounting for within-couple correlation). Each model will include fixed effects (separate for patient or partner) for group, month, group-by-month interactions, the baseline value of the outcome scale, baseline treatment type, number of baseline comorbidities, couple relationship quality, and demographics. Models will include random dyad and participant nested within dyad effects to account for within-dyad and within-person correlations between longitudinal responses. For the primary comparison, separately for each participant type, we will first test for any differences between groups across all 3 time points using an appropriately specified 3 degree of freedom linear contrast.

Secondary Aim: To test the effects of PERC on appraisals and coping resources, we will use similar models to compare each of the QOL subdomains groups, and to test the Secondary outcome hypotheses. We will explore the potential mediating effects of appraisal and coping resources using a longitudinal path analysis model.(70). The model will include all appropriate within-dyad and longitudinal correlations.

\section{Interim analyses $\{21 b\}$}

N/A

\section{Methods for additional analyses (e.g. subgroup analyses) \{20b\}}

Exploratory Aim: Determine if patient race/ethnicity, education, type of cancer treatment, and couples' relationship

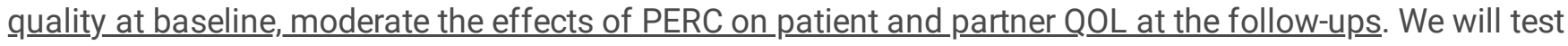
appropriate experimental group-by-moderator interactions using similar linear mixed models as specified for the Primary Aim. We will also analyze outcome and process data to identify critical characteristics of PERC participants, e.g., differences in racial/ethnic and education, in their PERC use patterns and outcomes.

\section{Methods in analysis to handle protocol non-adherence and any statistical methods to handle missing data $\{20 \mathrm{c}\}$}

We closely follow the regulatory documentation and reporting process that are strictly implemented by the $\mathrm{NIH}$ and UNC IRB for any non-adherence and deviations. We use the intent to treat methods to handle missing data. 
Plans to give access to the full protocol, participant level-data and statistical code $\{31 \mathrm{c}\}$

- The members of our research team will have access to the trial dataset that is deidentified once the trial is completed. The final dataset will be available for researchers who are interested in the related topics after the research team has disseminated the main findings of the research aims. Permission from the PI is required for any publications and dissemination effort.

\section{Oversight and monitoring}

Composition of the coordinating centre and trial steering committee $\{5 \mathrm{~d}\}$

N/A.

\section{Composition of the data monitoring committee, its role and reporting structure $\{21 \mathrm{a}\}$}

This study is a population-based, statewide randomized controlled efficacy trial evaluating a behavioral intervention in which prostate cancer patients who are post-initial treatment with curative intent and their partners use a web-based symptom self-management intervention at their homes to enhance their knowledge and skills, promote social support, facilitate health behaviors, and ultimately improve their QOL. A data monitoring committee board (DSMB) is not needed because this study is a minimal risk study. However, we have implemented a data and safety-monitoring plan for monitoring the trial with an executive committee of study leaders (PI and Co-ls) and study team members who implement study procedures to ensure the safety of participants as well as the validity and integrity of the data.

\section{Adverse event reporting and harms $\{22\}$}

An Adverse Event Monitoring Committee has also been formed to oversee the conduct of the study. Chaired by the PI, the committee is comprised of the Co-investigators. The committee meets regularly (monthly during the first two years of the study and then quarterly) to review the activities of the study. The research staff ensures all entry criteria are met prior to the initiation of the protocol. They also ensure that all study procedures and reporting of adverse events and unanticipated events is performed according to the IRB-approved protocol. All intervention-related adverse events will be reported to the IRB within 3-7 days. The PI will submit necessary reports to the funding agency. The PI and the Adverse Event Monitoring Committee assess the level of risk from adverse events (AEs) as mild (no interference in usual activities); moderate (some interference in usual activities); or severe (usual activities were significantly interrupted). The PI and the Adverse Event Monitoring Committee will assess whether events are related to the study using the following categories: not related, unlikely, possible, probable, or definite. We also hire an Independent Monitor who is independent from the present study design or implementation for data and safety monitoring. The Adverse Event Monitoring Committee will report adverse events to the Independent Monitor quarterly and when necessary to minimize research-associated risks.

\section{Frequency and plans for auditing trial conduct $\{23\}$}

The PI convenes weekly meetings with the research staff to review project progress, subject accrual, follow-up, and any anticipated or unanticipated AEs. The PI and project manager are responsible for monitoring study processes and ensuring that AEs and unanticipated events (UEs) are reported immediately to the Adverse Event Monitoring Committee, the Independent Safety Officer, and the IRB within 3-7 days of the incidents. Additionally, the PI will report to the funding agency at $\mathrm{NIH}$ within 24 hours the severe AEs and actions, if any, taken by investigators or the IRB because of the event or its continuing review. The study submits a complete data and safety monitoring summary report to the IRB as part of the annual renewal approval process and to the NIH with the annual progress report. 


\section{Plans for communicating important protocol amendments to relevant parties (e.g. trial participants, ethical committees) $\{25\}$}

See above

\section{Dissemination plans $\{31 \mathrm{a}\}$}

Dissemination has been considered in the design of PERC such as ease of use by participants and staff (with the possibility of removing the moderated online Forum after the efficacy testing. We are well positioned to disseminate the self-directed, eHealth PERC program by working with prostate cancer advocacy and support groups and by incorporating PERC into clinical care for men with prostate cancer and their partners. We will also disseminate the study results at national and international conferences and in peer-reviewed interdisciplinary journals.

\section{Discussion}

This study will provide a novel model for self-managing chronic illness symptoms that impact couples' relationships, intimacy, and quality of life. It addresses the National Institute of Nursing Research's goal to develop and test new strategies for symptom self-management to help patients and caregivers better manage their illness and improve quality of life. It also responds to calls for programs from the Institute of Medicine and American Cancer Society to address treatment-related effects and improve survivors' QOL.

\section{Trial status}

The recruitment for this RCT began May 30, 2018 and was completed April 30, 2021. We plan to complete the followup surveys by July $1^{\text {st }}, 2022$. The research team was busy with competing demands of daily operation of this R01 and other ongoing research projects before COVID-19 occurred in early 2020, which has significantly delayed all of our research activities, including publications.

\section{The study protocol date: May 28, 2020.}

\section{Abbreviations}

RCT: randomized clinical trial

QOL: quality of life

PERC: Prostate cancer resources for couples

$\mathrm{NIH}$ : National Institutes of Health

$\mathrm{AE}:$ adverse event

UE: unexpected event

IRB: Institutional review board

PI: Principal investigator

Co-I: Co-investigator 


\section{Declarations}

Ethics approval and consent to participate: IRB approval attached.

Consent for publication: we are willing to provide a model consent form on request once the study is completed.

Availability of data and materials: The members of our research team will have access to the trial dataset that is deidentified once the trial is completed. The final dataset will also be available for researchers who are interested in the related topics after the research team has disseminated the main findings of the research aims. Permission from the $\mathrm{PI}$ is required for any publications and dissemination effort.

Competing interests: none to disclose.

\section{Acknowledgements}

The University of North Carolina-Lineberger Comprehensive Cancer Center Cancer (UNC LCCC) Prevention and Control Intervention Research Pilot Grant Award (PI: Song) and UNC LCCC Population Sciences Developmental Research Award (PI: Song) have provided funding for the pilot testing of PERC. We'd like to thank the prostate cancer patients, their families, health care professionals, and our research staff for their contributions in the pilot studies and our ongoing trial to make the study possible.

\section{Authors' contributions $\{31 \mathrm{~b}\}$}

LS is the Principal Investigator; she conceived the study, led the proposal and protocol development, as well as the study implementation and data analyses.

MN contributed to study design, development of the proposal and study implementation.

RC contributed to study design, development of the proposal and study implementation EF was the lead trial methodologist.

CR contributed to study design, development of the proposal and study implementation

TK contributed to study design, development of the proposal and study implementation

El contributed to the protocol refinement and study implementation

GF contributed to the protocol refinement and study implementation

LN contributed to the study conceptualization, development of the proposal and the protocol.

MP RC contributed to study design, development of the proposal and study implementation EF was the lead trial methodologist.

XMT contributed to data analyses and the protocol development and implementation.

All authors read and approved the final manuscript."

\section{Funding \{4\}}


National Institute of Health National Institute of Nursing Research (R01 NR016990, PI: Song). NOA letter is attached.

\section{Availability of data and materials $\{29\}$}

The members of our research team will have access to the trial dataset that is deidentified once the trial is completed. The final dataset will be available for researchers who are interested in the related topics after the research team has disseminated the main findings of the research aims. Permission from the PI is required for any publications and dissemination effort.

\section{Ethics approval and consent to participate $\{24\}$}

We have received approval from the Institutional Review Boards at the University of North Carolina at Chapel Hill for this trial. The IRB approval document is attached.

\section{Consent for publication $\{32\}$}

we are willing to provide a model consent form on request once the study is completed

\section{Competing interests $\{28\}$}

none to disclose.

\section{Authors' information (optional)}

Opt out

\section{References}

1. American Cancer Society. Cancer Facts \& Figs. 2020. Atlanta, GA2020.

2. Siegel R, DeSantis C, Virgo K, Stein K, Mariotto A, Smith T, et al. Cancer treatment and survivorship statistics, 2012. Cancer J Clin. 2012;62(4):220-41.

3. Chen RC, Zhang Y, Chen MH, McMahon E, Loffredo M, McPherson CP, et al. Patient-reported quality of life during radiation treatment for localized prostate cancer: results from a prospective phase II trial. BJU Int. 2012;110(11):1690-5.

4. Matthew AG, Alibhai SM, Davidson T, Currie KL, Jiang H, Krahn M, et al. Health-related quality of life following radical prostatectomy: long-term outcomes. Quality of life research: an international journal of quality of life aspects of treatment care rehabilitation. 2014;23(8):2309-17.

5. Prabhu V, Lee T, McClintock TR, Lepor H. Short-. Intermediate-, and Long-term Quality of Life Outcomes Following Radical Prostatectomy for Clinically Localized Prostate Cancer. Rev Urol. 2013;15(4):161-77.

6. Crook JM, Gomez-Iturriaga A, Wallace K, Ma C, Fung S, Alibhai S, et al. Comparison of Health-Related Quality of Life 5 Years After SPIRIT: Surgical Prostatectomy Versus Interstitial Radiation Intervention Trial. J Clin Oncol. 2011;29(4):362-8.

7. van Tol-Geerdink JJ, Leer JWH, van Oort IM, van Lin EJNT, Weijerman PC, Vergunst H, et al. Quality of life after prostate cancer treatments in patients comparable at baseline. $\mathrm{Br} J$ Cancer. 2013;108(9):1784-9.

8. Bourke L, Smith D, Steed L, Hooper R, Carter A, Catto J, et al. Exercise for Men with Prostate Cancer: A Systematic Review and Meta-analysis. European urology. 2016;69(4):693-703. 
9. Lev E, Eller L, Gejerman G, Kolassa J, Colella J, Pezzino J, et al. Quality of life of men treated for localized prostate cancer: outcomes at 6 and 12 months. Support Care Cancer. 2009;17(5):509-17.

10. Reeve BB, Chen RC, Moore DT, Deal AM, Usinger DS, Lyons JC, et al. Impact of comorbidity on health-related quality of life after prostate cancer treatment: combined analysis of two prospective cohort studies. BJU international. 2014.

11. National Cancer Institute. SEER Cancer Statistics Factsheets: Prostate Cancer. U.S. National Institutes of Health: National Cancer Institute; 2018.

12. Epstein MM, Edgren G, Rider JR, Mucci LA, Adami HO. Temporal trends in cause of death among Swedish and US men with prostate cancer. J Natl Cancer Inst. 2012;104(17):1335-42.

13. Carneiro A, Sasse AD, Wagner AA, Peixoto G, Kataguiri A, Neto AS, et al. Cardiovascular events associated with androgen deprivation therapy in patients with prostate cancer: a systematic review and meta-analysis. World $\mathrm{J}$ Urol. 2015;33(9):1281-9.

14. Nguyen PL, Je Y, Schutz FA, Hoffman KE, Hu JC, Parekh A, et al. Association of androgen deprivation therapy with cardiovascular death in patients with prostate cancer: a meta-analysis of randomized trials. Jama.

2011;306(21):2359-66.

15. Edwards BK, Noone AM, Mariotto AB, Simard EP, Boscoe FP, Henley SJ, et al. Annual Report to the Nation on the status of cancer, 1975-2010, featuring prevalence of comorbidity and impact on survival among persons with lung, colorectal, breast, or prostate cancer. Cancer. 2014;120(9):1290 - 314.

16. Cheville AL, Kollasch J, Vandenberg J, Shen T, Grothey A, Gamble G, et al. A home-based exercise program to improve function, fatigue, and sleep quality in patients with Stage IV lung and colorectal cancer: a randomized controlled trial. J Pain Symptom Manag. 2013;45(5):811-21.

17. Mishra SI, Scherer RW, Geigle PM, Berlanstein DR, Topaloglu O, Gotay CC, et al. Exercise interventions on healthrelated quality of life for cancer survivors. Cochrane Database Syst Rev. 2012;8:Cd007566.

18. Kenfield SA, DuPre N, Richman EL, Stampfer MJ, Chan JM, Giovannucci EL. Mediterranean diet and prostate cancer risk and mortality in the Health Professionals Follow-up Study. European urology. 2014;65(5):887-94.

19. Lopez-Guarnido O, Alvarez-Cubero MJ, Saiz M, Lozano D, Rodrigo L, Pascual M, et al. Mediterranean diet adherence and prostate cancer risk. 2015(1699-5198 (Electronic)).

20. Moller E, Galeone C, Andersson TM, Bellocco R, Adami HO, Andren O, et al. Mediterranean Diet Score and prostate cancer risk in a Swedish population-based case-control study. Journal of nutritional science. 2013;2:e15.

21. Dinu M, Abbate R, Gensini GF, Casini A, Sofi F. Vegetarian, vegan diets and multiple health outcomes: a systematic review with meta-analysis of observational studies. Critical reviews in food science and nutrition. 2016:0.

22. Castro I, Waclawovsky G, Marcadenti A. Nutrition and physical activity on hypertension: implication of current evidence and guidelines. Current hypertension reviews. 2015;11(2):91-9.

23. Ervik B, Nordøy T, Asplund K. In the Middle and on the Sideline: The Experience of Spouses of Men With Prostate Cancer. Cancer Nursing. 2013;36(3):E7-E14 0.1097/NCC.0b013e31824fe1ef.

24. Northouse LL, Mood D, Montie JE, Sandler HM, Forman JD, Hussain M, et al. Living with Prostate Cancer: Patients' and Spouses' Psychosocial Status and Quality of Life. J Clin Oncol. 2007;25:4171-7.

25. Song L, Northouse LL, Braun TM, Zhang L, Cimprich B, Ronis DL, et al. Assessing longitudinal quality of life in prostate cancer patients and their spouses: a multilevel modeling approach. Quality of life research: an international journal of quality of life aspects of treatment care rehabilitation. 2011;20(3):371-81.

26. O'Brien R, Rose P, Campbell C, Weller D, Neal RD, Wilkinson C, et al. "I wish I'd told them": a qualitative study examining the unmet psychosexual needs of prostate cancer patients during follow-up after treatment. Patient

Page 15/19 
Educ Couns. 2011;84(2):200-7.

27. Hanly N, Mireskandari S, Juraskova I. The struggle towards 'the New Normal': a qualitative insight into psychosexual adjustment to prostate cancer. BMC Urol. 2014;14(1):56.

28. Letts C, Tamlyn K, Byers ES. Exploring the impact of prostate cancer on men's sexual well-being. J Psychosoc Oncol. 2010;28(5):490-510.

29. Galbraith ME, Fink R, Wilkins GG. Couples Surviving Prostate Cancer: Challenges in Their Lives and Relationships. Semin Oncol Nurs. 2011;27(4):300-8.

30. Kershaw T, Mood D, Newth G, Ronis DL, Sanda M, Vaishampayan U, et al. Longitudinal analysis of a model to predict quality of life in prostate cancer patients and their spouses. Ann Behav Med. 2008;36(2):117-28.

31. Hagedoorn M, Sanderman R, Bolks HN, Tuinstra J, Coyne JC. Distress in couples coping with cancer: a metaanalysis and critical review of role and gender effects. Psychol Bull. 2008;134(1):1-30.

32. Skolarus TA, Wolf AM, Erb NL, Brooks DD, Rivers BM, Underwood W 3. American Cancer Society prostate cancer survivorship care guidelines. Cancer J Clin. 2014;64(4):225-49. rd, et al.

33. National Comprehensive Cancer Network. NCCN Guidelines for Patients: Prostate Cancer. 2014.

34. NCCN Clinical Practice Guidelines in Oncology (NCCN Guideline). Prostate Cancer [Internet]. National Comprehensive Cancer Network; 2014.

35. Song L, Rini C, Deal AM, Nielsen ME, Chang H, Kinneer P, et al. Improving Couples' Quality Of Life Through A WebBased, Couple-Oriented Prostate Cancer Education Intervention. Oncol Nurs Forum. 2015;42(2):183-92.

36. Northouse LL, Mood DW, Schafenacker A, Montie JE, Sandler HM, Forman JD, et al. Randomized clinical trial of a family intervention for prostate cancer patients and their spouses. Cancer. 2007;110(12):2809-18.

37. Northouse LL, Mood D, Kershaw T, Schafenacker A, Mellon S, Walker J, et al. Quality of life of women with recurrent breast cancer and their family members. J Clin Oncol. 2002;20:4050-64.

38. Oncology Nursing Society. Putting evidence into practice. A pocket guide to cancer symptom management. Pittsburgh: Oncology Nursing Society; 2014.

39. Lazarus RS. Stress: appraisal and coping capacities. In: Eichler A, Silverman MM, Pratt DM, editors. National Institute of Mental Health: how to define and research stress. Washington, DC: American Psychiatric Press, Inc; 1984.

40. Lazarus RS, Folkman S. Stress, Appraisal, and Coping. New York: Springer Publishing Company; 1984.

41. Nagler RH, Gray SW, Romantan A, Kelly BJ, DeMichele A, Armstrong K, et al. Differences in information seeking among breast, prostate, and colorectal cancer patients: results from a population-based survey. Patient Educ Couns. 2010;81(Suppl):54-62.

42. Digital Readiness Gaps [Internet]. Pew Research Center. 2016. Available from:

http://www.pewinternet.org/2016/09/20/digital-readiness-gaps/.

43. Zickuhr K, Madden M. Older Adults and Internet Use. Pew Research Center's Internet \& American Life Project. Pew Internet \& American Life Project; 2012.

44. Older adults and technology use [Internet]. Pew Research Center. 2014. Available from: http://www.pewinternet.org/2014/04/03/older-adults-and-technology-use/.

45. Americans' IA: 2000-2015 [Internet]. Pew Research Center. 2016. Available from: http://www.pewinternet.org/2015/06/26/americans-internet-access-2000-2015/.

46. Bellg AJ, Borrelli B, Resnick B, Hecht J, Minicucci DS, Ory M, et al. Enhancing treatment fidelity in health behavior change studies: best practices and recommendations from the NIH Behavior Change Consortium. Health 
psychology: official journal of the Division of Health Psychology American Psychological Association. 2004;23(5):443-51.

47. Eaton LH, Doorenbos AZ, Schmitz KL, Carpenter KM, McGregor BA. Establishing treatment fidelity in a web-based behavioral intervention study. Nursing research. 2011;60(6):430-5.

48. Cella D, Tulsky D, Gray G, Sarafian B, Linn E, Bonomi A, et al. The Functional Assessment of Cancer Therapy scale: development and validation of the general measure. J Clin Oncol. 1993;11:570-9.

49. Esper P, Mo F, Chodak G, Sinner M, Cella D, Pienta KJ. Measuring quality of life in men with prostate cancer using the functional assessment of cancer therapy-prostate instrument. Urology. 1997;50(6):920-8.

50. Song L, Tan X, Bredle J, Bennett AV, Northouse L. Psychometric Properties of the FACT-G Quality of Life Scale For Family Caregivers of Cancer Patients. Quality of Life Research. 2020;In press.

51. Oberst M. Appraisal of Illness Scale: Manual for Use. Detroit: Wayne State University; 1991.

52. Lambert SD, Yoon H, Ellis KR, Northouse L. Measuring appraisal during advanced cancer: psychometric testing of the appraisal of caregiving scale. Patient Educ Couns. 2015;98(5):633-9.

53. Lewis FM. Family Home Visitation Study Final Report. Bethesda: National Cancer Institute, National Institutes of Health; 1996.

54. PROMISE Instrumental Support Instruments [Internet]. 2015 [cited April 12, 2016]. Available from:

https://www.assessmentcenter.net/documents/PROMIS\%20Instrumental\%20Support\%20Scoring\%20Manual.pdf.

55. PROMISE Emotional Support Instruments [Internet]. 2015 [cited April 12, 2016]. Available from:

https://www.assessmentcenter.net/documents/PROMIS\%20Instrumental\%20Support\%20Scoring\%20Manual.pdf.

56. PROMISE Informational Support Instruments [Internet]. 2015 [cited April 12, 2016]. Available from:

https://www.assessmentcenter.net/documents/PROMIS\%20Instrumental\%20Support\%20Scoring\%20Manual.pdf.

57. Sarason IGL, Henry M, Basham RB, Sarason BR. Assessing social support: The Social Support Questionnaire. J Pers Soc Psychol. 1983;44(1):127-39.

58. Jilcott SB, Keyserling TC, Samuel-Hodge CD, Johnston LF, Gross MD, Ammerman AS. Validation of a brief dietary assessment to guide counseling for cardiovascular disease risk reduction in an underserved population. J Am Diet Assoc. 2007;107(2):246-55.

59. Gardiner PA, Clark BK, Healy GN, Eakin EG, Winkler EA, Owen N. Measuring older adults' sedentary time: reliability, validity, and responsiveness. Med Sci sports Exerc. 2011;43(11):2127-33.

60. Van Cauwenberg J, Van Holle V, De Bourdeaudhuij I, Owen N, Deforche B. Older adults' reporting of specific sedentary behaviors: validity and reliability. BMC Public Health. 2014;14:734.

61. Wei JT, Dunn RL, Litwin MS, Sandler HM, Sanda MG. Development and validation of the expanded prostate cancer index composite (EPIC) for comprehensive assessment of health-related quality of life in men with prostate cancer. Urology. 2000;56(6):899-905.

62. PROMISE Pain Interference Instrument [Internet]. 2015 [cited April 12, 2016]. Available from: https://www.assessmentcenter.net/documents/PROMIS\%20Instrumental\%20Support\%20Scoring\%20Manual.pdf.

63. PROMISE Pain Intensity Instruments [Internet]. 2015 [cited April 12, 2016]. Available from: https://www.assessmentcenter.net/documents/PROMIS\%20Instrumental\%20Support\%20Scoring\%20Manual.pdf.

64. PROMIS Fatigue Instruments [Internet]. PROMIS. 2015 [cited April 12, 2016]. Available from: https://www.assessmentcenter.net/documents/PROMIS\%20Depression\%20Scoring\%20Manual.pdf.

65. PROMISE Sleep Disturbance Instruments [Internet]. 2015 [cited April 12, 2016]. Available from: https://www.assessmentcenter.net/documents/PROMIS\%20Instrumental\%20Support\%20Scoring\%20Manual.pdf. 
66. PROMIS anxiety instruments [Internet]. PROMIS. 2015 [cited April 12, 2016]. Available from:

https://www.assessmentcenter.net/documents/PROMIS\%20Anxiety\%20Scoring\%20Manual.pdf.

67. PROMIS Depression Instruments [Internet]. PROMIS. 2015 [cited April 12, 2016]. Available from:

https://www.assessmentcenter.net/documents/PROMIS\%20Depression\%20Scoring\%20Manual.pdf.

68. Littell RC, Milliken GA, Stroup WW, Wolfinger RD, Schabenberger O. SAS for mixed models [electronic resource]. Littell RC, editor Cary, N.C.: SAS Institute, Inc.; 2006.

69. Northouse LL, Rosset T, Phillips L, Mood DW, Schafenacker A, Kershaw T. Research with families facing cancer:

The challenges of accrual and retention. Res Nurs Health. 2006;29:199-211.

70. Kelloway EKa. Using Mplus for structural equation modeling: a researcher's guide. Los Angeles: SAGE; 2015.

\section{Figures}

\begin{tabular}{|c|c|c|c|c|c|c|c|c|}
\hline \multirow[b]{3}{*}{ TIMEPOINT** } & \multicolumn{8}{|c|}{ STUDY PERIOD } \\
\hline & \multirow{2}{*}{$\begin{array}{c}\text { Enrolment } \\
-t_{1} \\
\end{array}$} & \multirow{2}{*}{$\begin{array}{c}\text { Allocation } \\
0\end{array}$} & \multicolumn{5}{|c|}{ Post-allocation } & \multirow{2}{*}{$\begin{array}{c}\text { Close-out } \\
t_{x}\end{array}$} \\
\hline & & & $t_{1}$ & $t_{2}$ & $t_{3}$ & $t_{4}$ & etc. & \\
\hline \multicolumn{9}{|l|}{ ENROLMENT: } \\
\hline \multirow{2}{*}{$\begin{array}{l}\text { Eligibility screen } \\
\text { Informed consent }\end{array}$} & $X$ & & & & & & & \\
\hline & $\mathrm{X}$ & & & & & & & \\
\hline \multicolumn{9}{|l|}{$n / a$} \\
\hline Allocation & & $X$ & & & & & & \\
\hline \multicolumn{9}{|l|}{ INTERVENTIONS: } \\
\hline \multicolumn{9}{|l|}{ [Intervention A] } \\
\hline \multicolumn{9}{|l|}{ [Intervention B] } \\
\hline \multicolumn{9}{|l|}{$[N / A]$} \\
\hline \multicolumn{9}{|l|}{ ASSESSMENTS: } \\
\hline $\begin{array}{r}\text { [all outcome } \\
\text { variables] }\end{array}$ & $\mathrm{x}$ & & & & & & & \\
\hline $\begin{array}{r}\text { [List outcome } \\
\text { variables] }\end{array}$ & & & & $X$ & $X$ & $x$ & & \\
\hline $\begin{array}{r}\text { [antecedent } \\
\text { variables] }\end{array}$ & $x$ & & & $X$ & $x$ & $\mathrm{X}$ & & \\
\hline
\end{tabular}




\section{Figure 1}

Example template of recommended content for the schedule of enrolment, interventions, and assessments. * *Recommended content can be displayed using various schematic formats. See SPIRIT 2013 Explanation and Elaboration for examples from protocols. **List specific timepoints in this row. 